\title{
Finite-element simulation of a new two-dissipative mechanisms model for bulk medium-density polyethylene
}

\author{
J. KICHENIN, K. DANG VAN \\ Laboratoire de Mécanique des Solides, URA 317 CNRS, Ecole Polytechnique, Palaiseau \\ 91128 Cedex, France \\ K. BOYTARD \\ Gaz de France, DETN, 361, avenue du Président Wilson, BP33, La Plaine Saint-Denis 93211, \\ France
}

A two-dissipative mechanisms model, associating a Maxwell and an elastoplastic model in parallel, is discussed in order to account for the non-linear viscoelasticity of bulk medium-density polyethylene. On the one hand, the experimental determination of the constitutive equations coefficients is described from a tensile specimen machined from gas pipes. On the other hand, finite-element simulation of the stress relaxation experiment, proposed by Sweeney and Ward, is achieved, which yields a complete analysis of the dissipative mechanisms interaction during the test. The finite-element code built upon this modelling is finally used in a tentative simulation of a cyclic pressure test on a pipe specimen.

\section{Introduction}

Predicting the answer to successive loads applied to polyethyleme structures presents a critical interest im a wide field of applications, including gas or watter pipes design The key problems are, on the one hand, to describe satisfactorily the constitutive equations of the materiall, which implies taking account of stress relaxation, geometricall recovery and straim-rate effects, and on the other hand, to study real structures, and not only one-dimensional temsile specinems, which requires the avallability of numerical tools, such as finite-element codes, providing am easy access to the predictions of the constitutive equations.

The purpose of this work was to introduee an alternative two-mechanisms model to the classical approaches based on viscoelasticity, such as that of Sweeney and Ward [1], in order to give am interpretation of the cyclie behaviour of polyethyleme samplex, and to derive a computational method for polyethylleme structures Professor Ward and collaborators put in evidence that a model based on a pair of thermally activated Maxwell models (Fig-1) xceurately represents creep and stress-reliaxation phemonema for hightmodiulus polyethyllene fibres Moreover, Swetemey amd Ward [1] demonstrated the seperionity of this approach to that of Lefebve and Escaig [2], brased on a umique thermally activated Mawwell model, when amallysing the response of oniented polyethylene fibres subinitted to a sequence of loading im two particular cases.

(i) The step stress relaxation experiment (Fig. 2): a tensile specinnen is rapidly loaded to a stress
$\sigma_{0}+\Delta \sigma_{\text {, where }} \Delta \sigma \ll \sigma_{0}$, and the strain then is freld constant whille the stress is allowed to decay to the value $\sigma_{\oplus,}$ at which poimt the speciumem is rapidly reloaded to the stress $\sigma_{\circledast}+\Delta \sigma_{\text {, }}$ and agaim allowed to relax, and so on The diecaly timme bettween the $i$ th and $(i+1)$ th lowaling step is wittem as $\Delta t_{i}$. The above authors experimentinility sted thati the sequence of $\Delta t_{i}$ increases, batt the sequence of rattios $\Delta t_{i+1} \mathrm{t} / \Delta t_{i t}$ tendis to one.

(iii) The step relaxathiom amd recovery expeniment (Fig. 3)) the previoush described test is internupted and followed by a rapied decrease of the imposed strain untill strows level $\sigma_{11}$ is reachedi Stress. recovery is then observed untill the wallue $\sigma_{1}+\Delta \sigma_{1}$ is attaineri, where a mew strain decnease camses the strexs: to take again the valine $\sigma_{1}$, amd amother recowery is allowed, and so on. Ar analycical standy of thins expeniment led the two authors to more simmitain resullts concerning sequences $\Delta t_{i}$ and $\Delta t_{i t+1} \mathrm{v} / \Delta \|_{i i}$ thim im the step stress relaxation experinmemt. The Lefebre amdi Escaig theory was incomsistemite with thiat wery poinat.

We propose a mortel asssociating a simple Maxwell modell and an elastwoplastic model with kimematic hardeming (Fig. 4)) The intrordaction of an elastoplas-

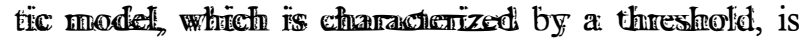
necessary to accoumet for the existence of residual stress, which cam be obsed in polyethyleme gas pipes, for exammple were elasstoplastic effects inexistent, these streses sfrould completely decay after some time. Thuss, the diffentences compared with the theory of Sweeney and Ward lies first in substituting an 


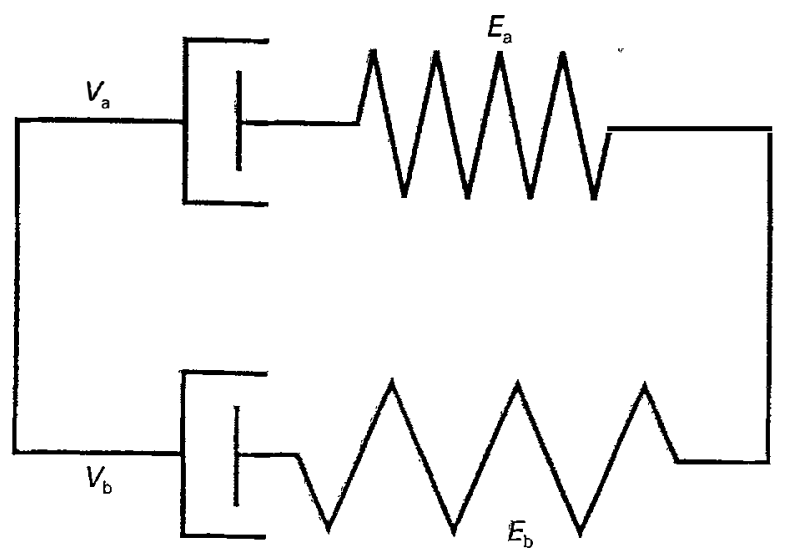

Figure 1 The two-process Sweeney and Ward model.

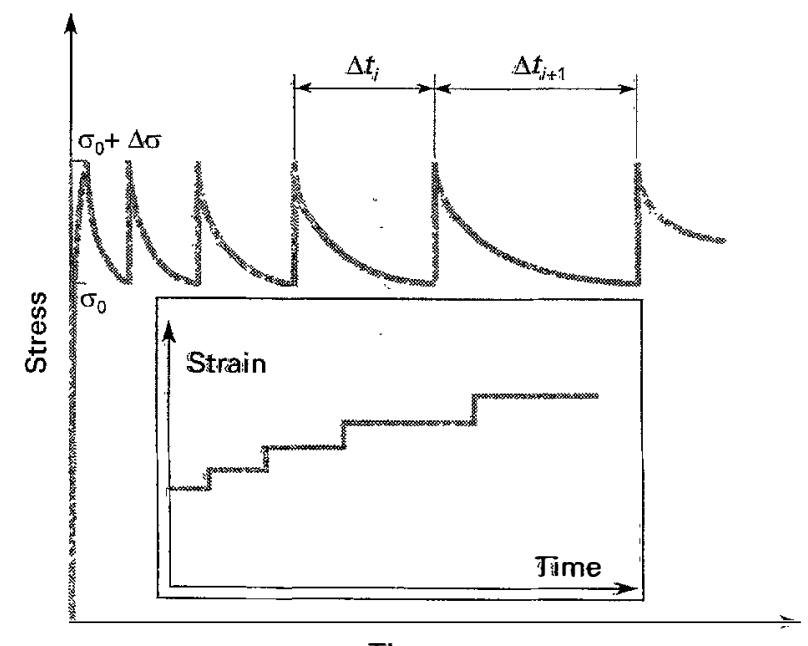

Time

Figure 2 The step stress relaxation experiment.

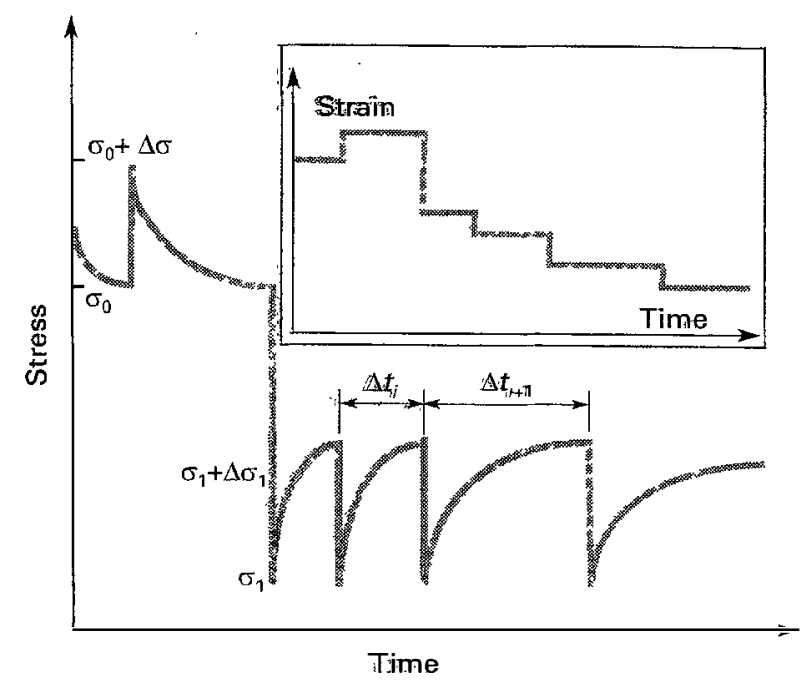

Figure 3 The stress relaxation and recovery experiment.

elastoplastic mechanism to a Maxwell one, and second in disregarding thermal activation processes for the sake of simplicity. Our theory is inspired from the study of bulk medinaromsity polyethylene samples obtained from extruded gas pipes. We intended to show that, on the one hand, it is consistent with

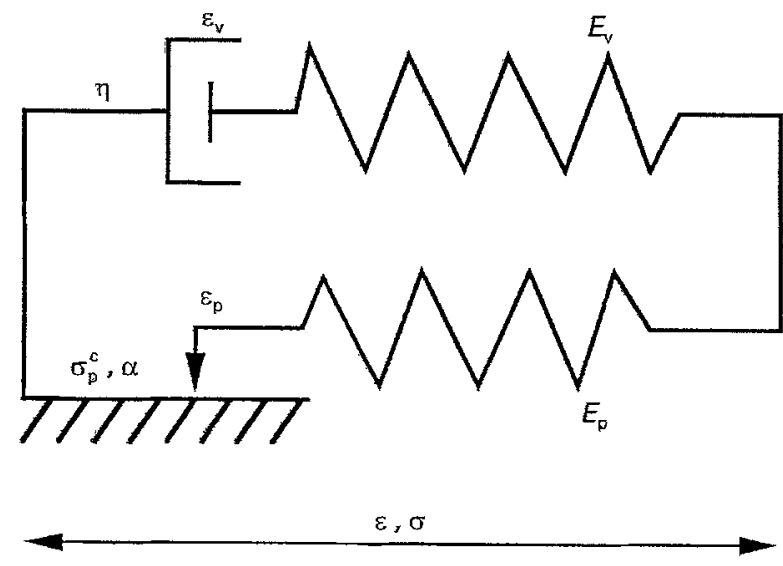

Figure 4 Coefficients of the proposed model.

Sweeney and Ward's conclusions concerning the step stress relaxation experiment, and the stress relaxation and recovery one, and on the other hand, that it enables discussion of a step pressure experiment realized on tube segments, for which a finite-element simulation was achieved. But first, we will detail some properties of the model and specify a route to identify experimentally the coefficients thus introduced.

\section{Model analysis}

It is necessary to describe an experimental procedure which enables a unique determination of coefficients in order to assert that the model is a reliable and useful tool for engineers. Coefficients of a constitutive equation provide indeed basic data to compare properties of materials, so testing organization is important.

Our models introduces five coefficients: modulus, $E_{\mathrm{v}}$, and viscosity; $\eta$, for the Maxwell mechanism, modulus, $E_{\mathrm{p}}$, threshold, $\sigma_{\mathrm{p}}^{\mathrm{c}}$, and a coefficient for kinematic hardening, $\alpha$, for the elastoplastic mechanism. We realized a series of traction-relaxation-geometrical recovery tests detailed below, in order to evaluate these parameters. We used a classical tensile specimen (Fig. 5) machined from a gas pipe wall, its main axis corresponding to the extrusion direction. Experimental apparatus consisted in an Instron press with a $500 \mathrm{~kg}$ load cell, an RDP transducer with range " $\pm 5 \mathrm{~mm}$, and an automatic data acquisition software agnes achieved in the LMS and running on a PC. The traction-relaxation-geometrical recovery test simultaneously takes into account strain versus time and stress versus time evolutions (Figs 6 and 7), and consists of three steps:

(i) choose a strain rate, $\dot{\varepsilon}_{\text {imp }}$, and a strain level, $\varepsilon_{\text {imp }}$, and realize a traction until the selected strain value is reached; $\sigma_{\max }$ denotes the maximum value of stress recorded;

(ii) hold the strain and allow the stress to relax during a period $\Delta t_{\text {rel }}$, at the end of which further evolution of the stress may be estimated to be negligible. Note the ultimate value $\sigma_{\text {lim }}$;

(iii) dismount the specimen from the press without removing the displacement captor (some reduction of the imposed strain may help) and observe the 

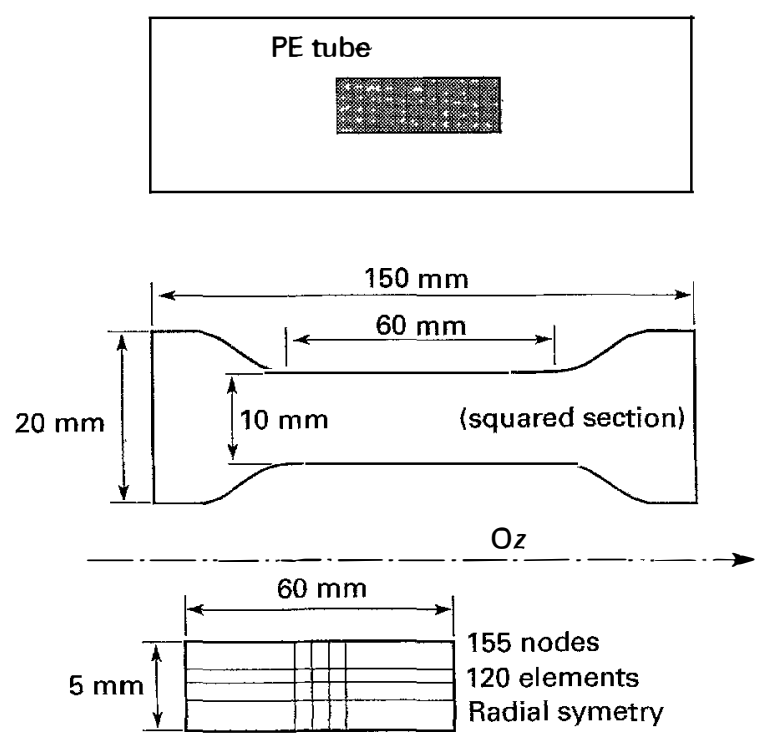

Figure 5 The tensile specimen and finite-element mesh.

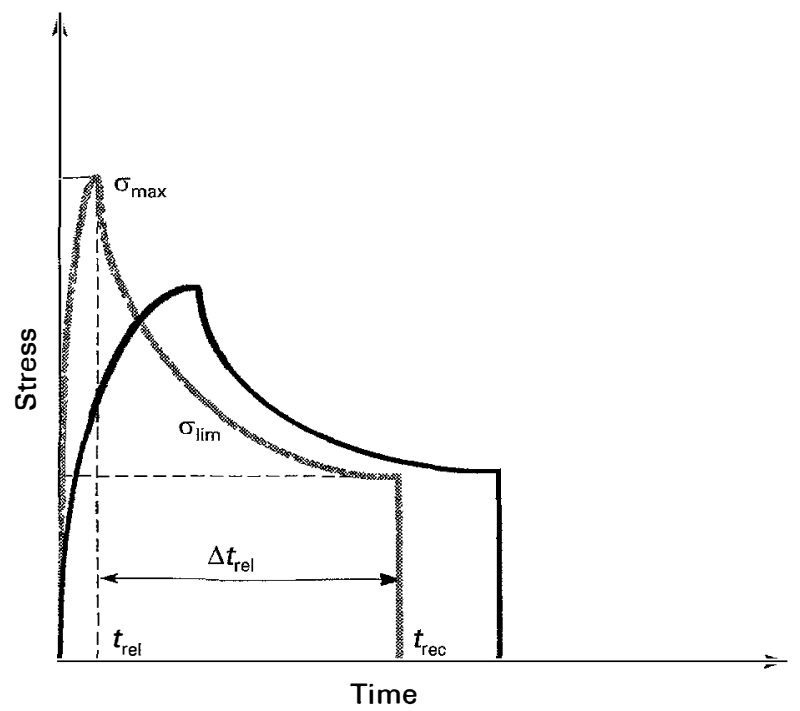

Figure 6 Stress versus time curve during the three-step experiment ${ }^{-}$ for different values of $\dot{\varepsilon}_{\text {imp }}$.

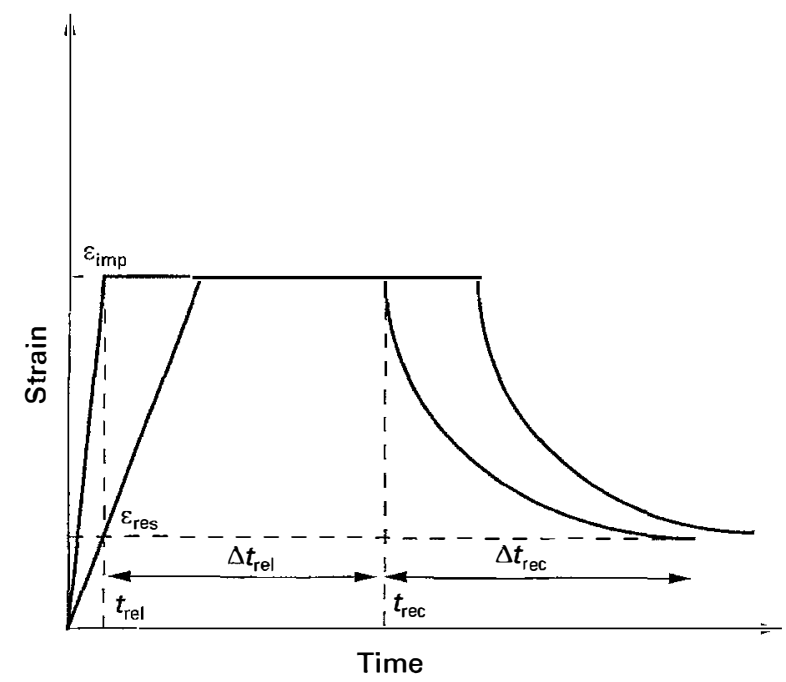

Figure 7 Strain versus time curve during the three-step experiment for different values of $\dot{\varepsilon}_{\text {imp }}$. geometrical recovery during a period $\Delta t_{\mathrm{rec}}$. So the specimen size reduces quickly first, then slowly, and stabilizes when $\Delta t_{\mathrm{rec}}$ is large enough, showing evidence of some unrecoverable elongation at room temperature, corresponding to a residual strain, $\varepsilon_{\text {res }}$.

Thus described, the test associates $\sigma_{\text {lim }}$ and $\varepsilon_{\text {res }}$ to $\varepsilon_{\text {imp }}$. One remarkable point is that the use of an initial strain rate ten times greater or lower than $\dot{\varepsilon}_{\text {imp }}$ scarcely modifies those values but, of course, affects the maximal stress recorded, $\sigma_{\max }$, at the end of the tensile step. We found it convenient, for our material, to conduct the experiment with the values $\Delta t_{\text {rel }}=24 \mathrm{~h}$, $\Delta t_{\text {rec }}=24 \mathrm{~h}$ and $\dot{\varepsilon}_{\text {imp }}=0.250 \times 10^{-2} \mathrm{~s}^{-1}$, while we also tried $0.025 \times 10^{-2} \mathrm{~s}^{-1}$ without noticeable change. Fig. 8 shows the stress versus time relaxation step for both strain rates when $\varepsilon_{\text {imp }}=15 \%$, and Fig. 9 the following geometrical recovery step. As an example of the series of measures to be followed in order to identify the coefficients, Fig. 10 shows the stress versus time evolution for various values of $\varepsilon_{\text {imp }}$ yielding various values of $\sigma_{\text {lim }}$, and Fig. 11 is the recovery step with values of $\varepsilon_{\text {res }}$ as an issue. Those data enable the drawing of $\sigma_{\text {lim }}$ versus $\varepsilon_{\text {imp }}$ curve and $\varepsilon_{\text {res }}$ versus $\varepsilon_{\text {imp }}$ curves, as in Figs 12 and 13, on the basis of which the determination of the coefficients is done.

To retain information from those drawings, one must keep in mind that a parallel association of two mechanisms simply sums their individual properties.

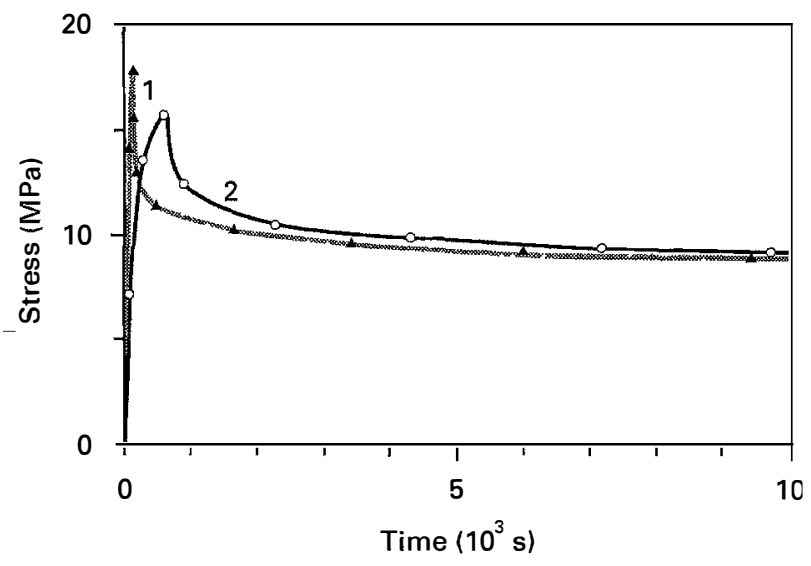

Figure 8 Measured stress relaxation over $3 \mathrm{~h}$.

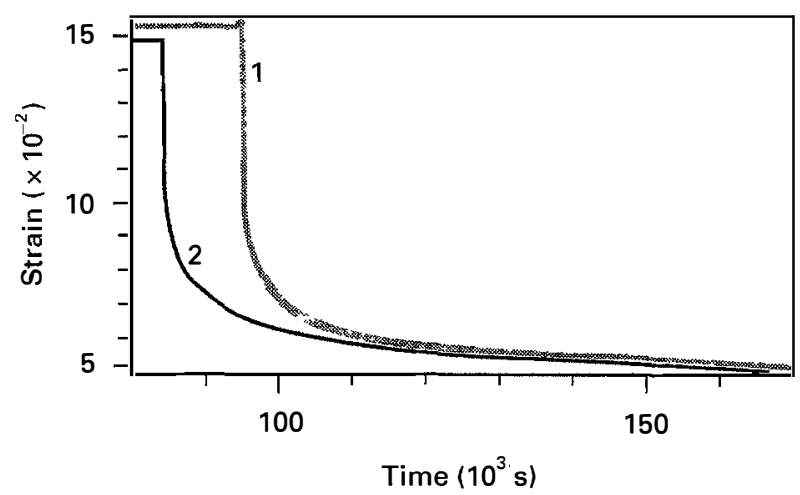

Figure 9 Measured geometrical recovery over $24 \mathrm{~h}$. 


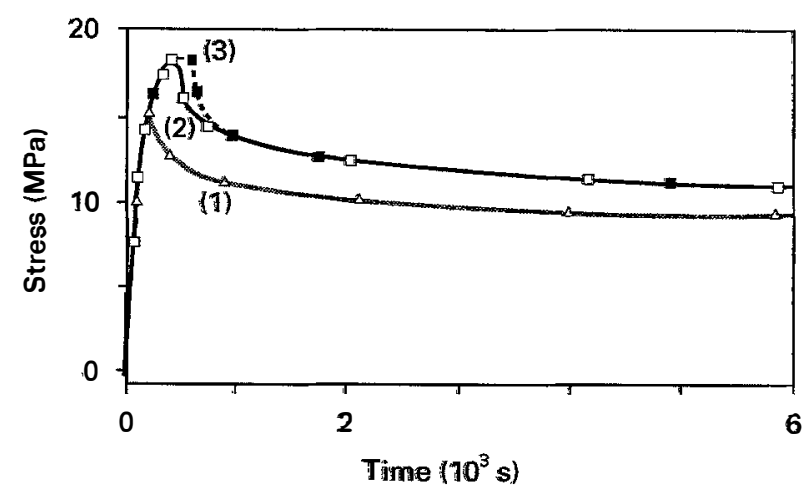

Figure 10 Stress relaxation occuring over more than $1 \mathrm{~b}$ for $\dot{\varepsilon}_{\mathrm{imm}}=$ $0.250 \times 10^{-2} \mathrm{~s}^{-1}$ and (1) $\varepsilon_{\mathrm{impp}}=0.08$, (2) $\varepsilon_{\mathrm{imp}}=0.115$, (3) $\varepsilon_{\mathrm{imp}}=0.15$.

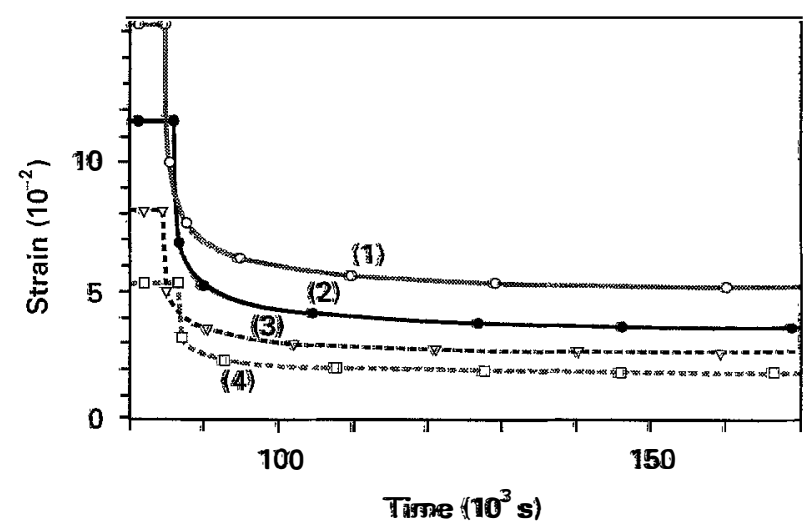

Figune 11 Strain recovery over $24 \mathrm{~h}$ for (1) $\varepsilon_{\text {ivomp }}=0.15$, (2) $\varepsilon_{\text {impo }}=$ $0.115,(3) \varepsilon_{\text {imp }}=0.08$ and (4) $\varepsilon_{\text {imp }}=0.05$.

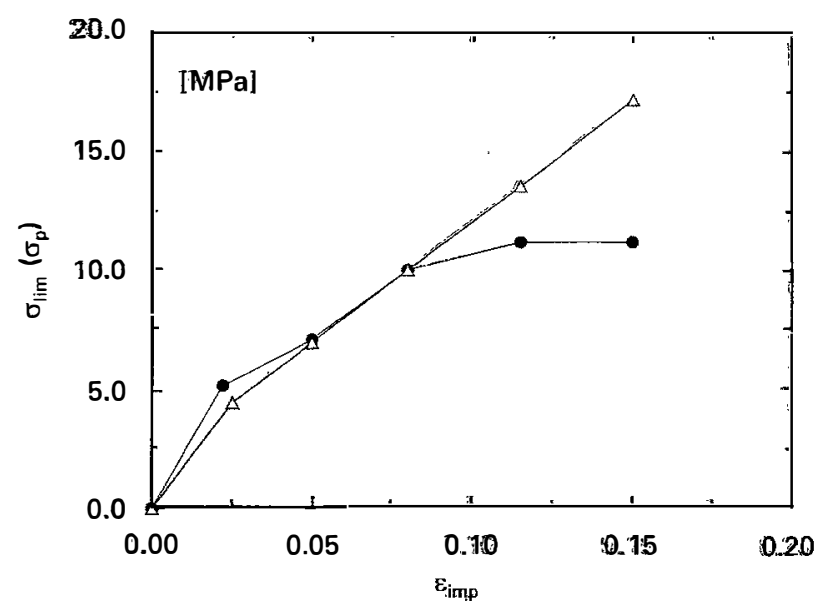

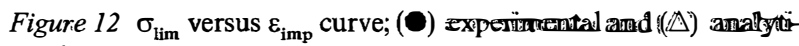
cal data.

If the constitutive equation for the Maxwell model is

$$
\begin{aligned}
\sigma_{\mathrm{v}} & =\eta \dot{\varepsilon}^{\mathrm{v}} \\
& =E_{\mathrm{v}}\left(\varepsilon-\varepsilon^{\mathrm{w}}\right)
\end{aligned}
$$

and for the elastoplastic mechanisisn with limear kinematic hardening (Fig. 14)

$$
\begin{aligned}
\sigma_{\mathrm{p}}= & E_{\mathrm{p}} \varepsilon \quad \text { if } \sigma_{\mathrm{p}}<\sigma_{\mathrm{p}}^{\mathfrak{c}} \\
\sigma_{\mathrm{p}}= & E_{\mathrm{p}}\left(\varepsilon_{\mathrm{c}}+g\left(\varepsilon-\varepsilon_{\mathrm{c}}\right)\right) \\
& \text { if } \sigma_{\mathrm{p}} \geqslant \sigma_{\mathrm{p}}^{\mathrm{c}} \text { witth } \varepsilon_{\mathfrak{c}}=\frac{\sigma_{\mathrm{p}}^{\mathrm{c}}}{E_{\mathrm{p}}}
\end{aligned}
$$

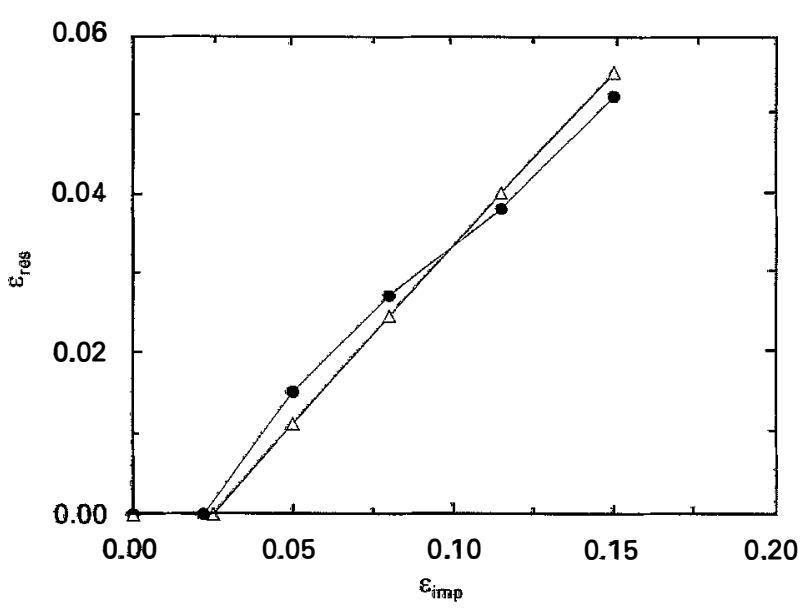

Figure $13 \varepsilon_{\text {res }}$ versus $\varepsilon_{\text {imp }}$ carve; $(\bullet)$ experimental and $(\triangle)$ analytical data.

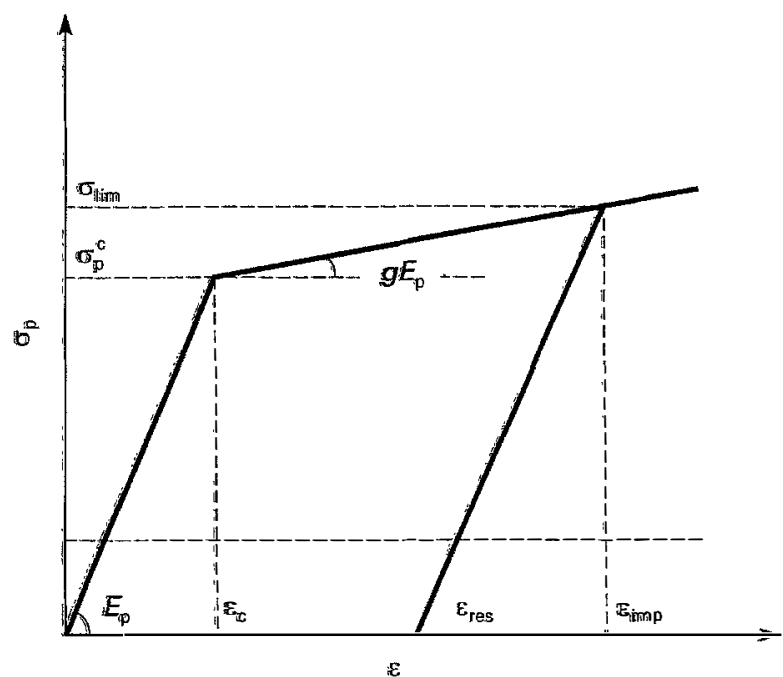

Figure 14 Stress versus strain carwe for the elastoplastic mechamism.

the constitutive equation for our model reads

$$
\sigma=\sigma_{\mathrm{w}}+\sigma_{\mathrm{p}}
$$

Assuming that the mechanissms are independent (this point will be discussed later), the relaxation step and the geometrical recovery step isolate the elastoplastic mechanasm part of the behaviour: after relaxation, this very mechanism withstands the overall stress, and after geometrical recowery, the ewentual residual strain is comnected to some moxement of the slide. So we

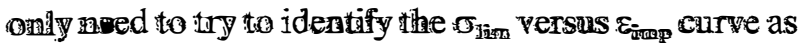
a classical stress strain curve for a tensile specimen, and consider it as the effect of an elastoplastic constitutive equation in the case of kisematic hardening. The determination of $E_{p}$ and $\sigma_{p}^{c}$ is then quite usual from the initial slope and the slope change. The coeffreient $\alpha$ is deduced frow the second slope by

$$
a=\frac{\mathfrak{g E} E_{\mathfrak{p}}}{1-g}
$$

The $\varepsilon_{\text {res }}$ wersus $\varepsilon_{\text {imp }}$ cinrve dinust prowe to be consistent With the prewious one consider a poind with coordinates 
$\left(\varepsilon_{\text {imp }}, \sigma_{\text {lim }}\right)$ and imagine an elastic unloading, i.e. draw a segment with slope $E_{\mathrm{p}}$ from this point to the strain axis. The point reached indicates the value $\varepsilon_{\text {res }}$ that should be read on the $\left(\varepsilon_{\text {imp }}, \varepsilon_{\text {res }}\right)$ diagram (Fig. 14).

The determination of $E_{\mathrm{v}}$ and $\eta$ is easier now that the other coefficients are known. The choice of a simple Maxwell model without any thermal activation naturally leads us to evaluate a relaxation time, $\tau$

$$
\frac{1}{\tau}=\frac{E_{\mathrm{v}}}{\eta}
$$

which is a rough approximation of the physical reality; we are aware of this. We deduce that the concept of a time-relaxation spectrum captures more precisely the complexity of polymer behaviour in this field, see, for example, Hadley and Ward [3]. However, this assumption retains enough simplicity within our model, to complete the investigation of its properties, and enable numerical simulations, which was our initial goal. So let us consider one of the relaxation curves and treat it like an exponential stress decay.

$$
\sigma=\exp \left(-\frac{t-t_{\mathrm{rel}}}{\tau}\right)\left(\sigma_{\max }-\sigma_{\lim }\right)+\sigma_{\mathrm{lim}}
$$

which yields $\tau$ when

$$
\sigma=\frac{\sigma_{\max }-\sigma_{\text {lim }}}{e}+\sigma_{\text {lim }}
$$

Our experimental procedure withdraws much information from the fully relaxed state, which in counterpart does not enable an easy determination of $E_{\mathbf{v}}$ straight from the measurements. We have to find $E_{\mathrm{v}}$ before an evaluation of its value from the recorded data, and then refine it with the help of a numerical software.

The previous evaluation is done on the basis of a test for which we suppose $\sigma_{\text {lim }}=\sigma_{\mathrm{p}}^{\mathrm{c}}$ and then estimate

$$
\sigma_{\max }=E_{\mathrm{v}} \varepsilon_{\mathrm{imp}}+\sigma_{\mathrm{p}}^{\mathrm{c}}
$$

which yields an approximation of $E_{\mathrm{v}}$. If numerical simulations of the tensile step of the test are achieved, and $E_{\mathrm{v}}$ is allowed to vary slightly, it is possible to fit the data satisfactorily and to select the desired value. This was achieved with our finite-element code, but any software taking into account Equations 1 and 2 could help in this task.

Finally, the values of the obtained parameters corresponding to our medium-density extruded polyethylene are $E_{\mathrm{p}}=175.0 \mathrm{MPa}, \sigma_{\mathrm{c}}^{\mathrm{p}}=4.375 \mathrm{MPa}$, $\alpha=245.6 \mathrm{MPa}, E_{\mathrm{v}}=65.0 \mathrm{MPa}, \eta=280.0 \mathrm{GPas}$.

One can now observe that our theory quantifies both plastic and viscous phenomena, very well known in the study of polymers. The elastoplastic model appears particularly well adapted to give an account of the memory effects of the material. We would like to point out that it is difficult to identify the parameters of our model just from a tensile test, or a creep test. The modelling just attempts to use the total diversity of experimental resources to capture the non-linear properties of the material.

To illustrate this, let us briefly discuss the coupling of the elastoplastic and the viscous mechanisms. It is experimentally evinced by modifying the second step of our procedure and by setting $\Delta t_{\text {rel }}$ to zero. So when the geometrical recovery phase immediately follows the traction, a reduction of the $\varepsilon_{\text {res }}$ values recorded is stated. To explain this, one may imagine that the chain reorganization occurring during relaxation influences further geometrical evolution of the specimen, which reveals some aspects of the versatile memory effects of the material [4]. Therefore, it is unnecessary to describe the stated coupling with an explicit relationship between internal parameters: when writing the generalization of Equations 1 and 2 in order to build a finite-element algorithm, this coupling becomes implicit from the equations of statics. We expand the details in another paper.

\section{Analytical validation}

Analytical expressions simulating the three-step experiment discussed in the previous section, can easily be derived from Equations 1 and 2. Let us suppose that $\dot{\varepsilon}_{\text {imp }}$ and $\varepsilon_{\text {imp }}$ are known; then

(i) the traction step: for the Maxwell mechanism

$$
\sigma_{\mathrm{v}}=\eta \dot{\varepsilon}_{\mathrm{imp}}\left[1-\exp \left(-\frac{E_{\mathrm{v}} \varepsilon_{\text {imp }}}{\eta \dot{\varepsilon}_{\text {imp }}}\right)\right]
$$

and for the elastoplastic mechanism, assuming $\sigma_{\mathrm{p}} \geqslant \sigma_{\mathrm{p}}^{\mathrm{c}}$

$$
\sigma_{\mathrm{p}}=\frac{\alpha}{\alpha+E_{\mathrm{p}}} E_{\mathrm{p}} \varepsilon_{\mathrm{imp}}+\frac{E_{\mathrm{p}}}{\alpha+E_{\mathrm{p}}} \sigma_{\mathrm{p}}^{\mathrm{c}}
$$

thus, with these values, $\sigma_{\max }=\sigma_{\mathrm{v}}+\sigma_{\mathrm{p}}$;

(ii) relaxation step: the Maxwell mechanism decreases to zero, following

$$
\sigma_{\mathrm{v}}=\eta \dot{\varepsilon}_{\mathrm{imp}}\left[\exp \left(\frac{E_{\mathrm{v}} \varepsilon_{\mathrm{imp}}}{\eta \dot{\varepsilon}_{\mathrm{imp}}}\right)-1\right] \exp \left(-\frac{E_{\mathrm{v}} t}{\eta}\right)
$$

and the elastoplastic mechanism is steady, yielding $\sigma_{\text {lim }}$

$$
\begin{aligned}
\sigma_{\mathrm{lim}} & =\sigma_{\mathrm{p}} \\
& =\frac{\alpha}{\alpha+E_{\mathrm{p}}} E_{\mathrm{p}} \varepsilon_{\mathrm{imp}}+\frac{E_{\mathrm{p}}}{\alpha+E_{\mathrm{p}}} \sigma_{\mathrm{p}}^{\mathrm{c}}
\end{aligned}
$$

(iii) recovery step: we must observe that during this step the overall stress is zero

$$
\sigma=\sigma_{\mathrm{v}}+\sigma_{\mathrm{p}}=0
$$

At the end of this step, $\sigma_{v}=0$.

The elastoplastic mechanism unloads elastically with a slope $E_{\mathrm{p}}$, thus residual strain is identified as

$$
\begin{gathered}
E_{\mathrm{p}}\left(\varepsilon_{\mathrm{imp}}-\varepsilon_{\mathrm{res}}\right)=\sigma_{1 \mathrm{im}} \\
\varepsilon_{\mathrm{res}}=\frac{E_{\mathrm{p}} \varepsilon_{\mathrm{imp}}-\sigma_{\mathrm{p}}^{\mathrm{c}}}{\alpha+E_{\mathrm{p}}}
\end{gathered}
$$

Analytical values of $\sigma_{\max }, \sigma_{\text {lim }}, \varepsilon_{\text {res }}$ are estimated from the data given in Section 2, and are reported in Figs 12 and 13 , where they compare satisfactorily with experimental data.

These expressions have been used to check the accuracy of the numerical results obtained by a prototype two-dimensional finite-element code. Our purpose is 
to validate definitely the code and then compute structures such as polyethylene gas pipes.

\section{Step stress relaxation simulation}

In order to illustrate the interest of the modelling and the numerical tool, which allows an easy investigation of the evolution of a phenomenon through variations of the coefficients, we first treat a one-dimensional case: the step stress relaxation experiment. We thus simulate loading upon an axisymmetric $6 \times 10^{-2} \mathrm{~m}$ long rod and $0.5 \times 10^{-2} \mathrm{~m}$ in radius, and only need to mesh a rectangle of these dimensions, representing a meridian section, with 120 linear rectangular elements (Fig. 5). Instructions given to perform the computation are quite close to the experimental practice: the operator specifies $\sigma, \Delta \sigma, \dot{\varepsilon}_{\text {imp }}$ and leaves the rest to the computer.

We investigate here one conclusion drawn by Sweeney and Ward [1] from the experimental study of step stress relaxation of high-modulus oriented fibres. It is concerned with the role of each mechanism during the test: it is hinted here that, at the beginning, the viscous mechanism part is predominant in the overall stress; then the successive relaxation steps cause this part to decay, while the elastoplastic mechanism component in the real stress smoothly increases. There is a process of transfer of stress between the mechanisms.

This leads to the simulation for two sets of coefficients.

(i) The first one corresponds to a material associated with the data: $E_{\mathrm{p}}=130.0 \mathrm{MPa}, \sigma_{\mathrm{c}}^{\mathrm{p}}=$ $7.05 \mathrm{MPa}, \quad \alpha=1.06 \mathrm{MPa}, \quad E_{\mathrm{v}}=65.0 \mathrm{MPa}, \quad \eta=$ 280.0 GPas.

We then chose $\sigma_{0}=9.0 \mathrm{MPa}$ and $\Delta \sigma=1.0 \mathrm{MPa}$, and $\dot{\varepsilon}_{\text {irnp }}=0.83 \times 10^{-2} \mathrm{~s}^{-1}$. In Fig. 15 we state how the plastic mechanism will step-by-step hold the imposed value of $\sigma_{0}$ while the viscous mechanism shows a decreasing evolution. Nevertheless, the viscous mechanism exhibits a lower contribution to the global stress than the elastoplastic one, even from the begin-

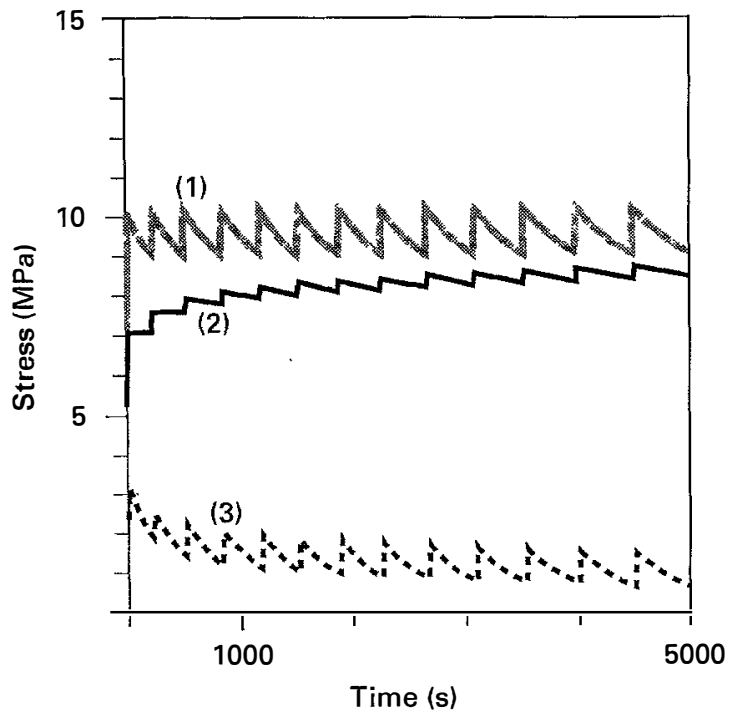

Figure 15 First set of coefficients: computed breakdown of the stress in the step relaxation experiment: (1) overall stress $\sigma_{z z}$, (2) elastoplastic mechanism part, $\sigma_{\mathrm{p} z z},(3)$ viscous part $\sigma_{\mathrm{v} z z}$. ning of the experiment, which marks a difference with the analysis reported below. In our modelling, the values of the coefficients definitely influence the ratio of the contribution of each mechanism in the apparent stress, which will be confirmed by the next example. It may be noticed that the ratio $E_{\mathrm{v}} / E_{\mathrm{p}}$ is here close to 0.5 . Fig. 16 shows the calculated increase of the strain; we unfortunately cannot produce any comparative measurements, because controlling the evolution of the stress between $\sigma_{0}$ and $\Delta \sigma$ indeed requires a sophisticated electronic apparatus. We should remark that a real specimen cannot withstand a level of strain greater than 0.30 without the occurrence of necking - this event is not predicted by the code - and there is a limit to the accuracy of the simulation. However, the number of oscillations found appears satisfactory compared with what can be evaluated from rough experiments. Fig. 17 provides evidence for the threedimensional properties of the modelling: the radial components of the stresses associated with each

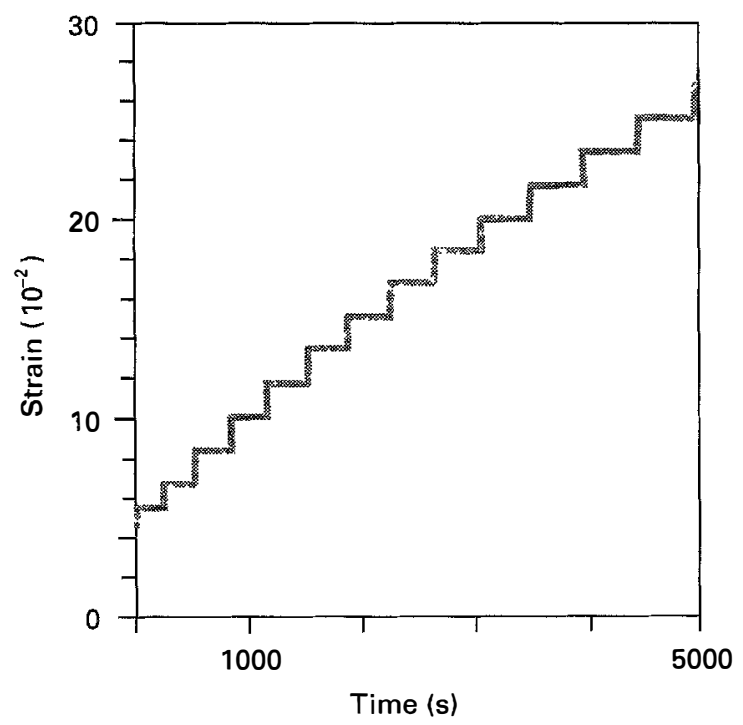

Figure 16 First set of coefficients: strain evolution.

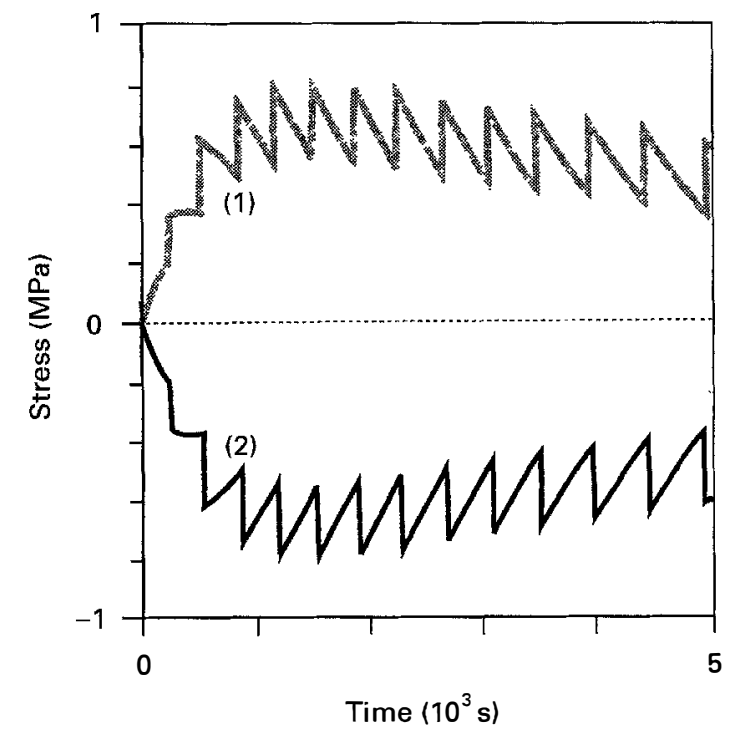

Figure 17 First set of coefficients: radial components, (1) elastoplastic mechanism part $\sigma_{\mathrm{prr}}$ and (2) viscous part $\sigma_{\mathrm{v} r}$. 
mechanism, $\sigma_{\mathrm{prr}}$ and $\sigma_{\mathrm{vrr}}$, take opposite values, demonstrating that the numerical computation introduces non-trivial effects in the plane perpendicular to the tensile axis.

(ii) The second set of coefficients that we tried was partly inspired from Sweeney and Ward's Table I [1], that is, we aimed to simulate some material with properties close to high-modulus oriented polyethylene, although we did not test such a specimen. We thus adopted $E_{\mathrm{v}}=28.0 \mathrm{GPa}$ and $E_{\mathrm{p}}=2.5 . \mathrm{GPa}$ and more arbitrarily fixed $\tau_{\mathrm{c}}=150.7 \mathrm{MPa}, \alpha=1.0 \mathrm{MPa}$, $\eta=140.0$ GPas. Strain rate is the same as in the first case. We also chose $\sigma_{0}=170.0 \mathrm{MPa}$ and $\Delta \sigma=$ $30.0 \mathrm{MPa}$. Results in Fig. 18 are consistent with the previously reported analysis, i.e. the plastic mechanism plays the part of a slow decay-rate mechanism, and initially bears lower stress level, while at the end it takes the whole overall value. The ratio $E_{\mathrm{v}} / E_{\mathrm{p}}$ is here 11.2 , and our model conforms to the scheme drawn by Sweeney and Ward, on a qualitative point of view. Quantitatively, it may seem odd that the simulation

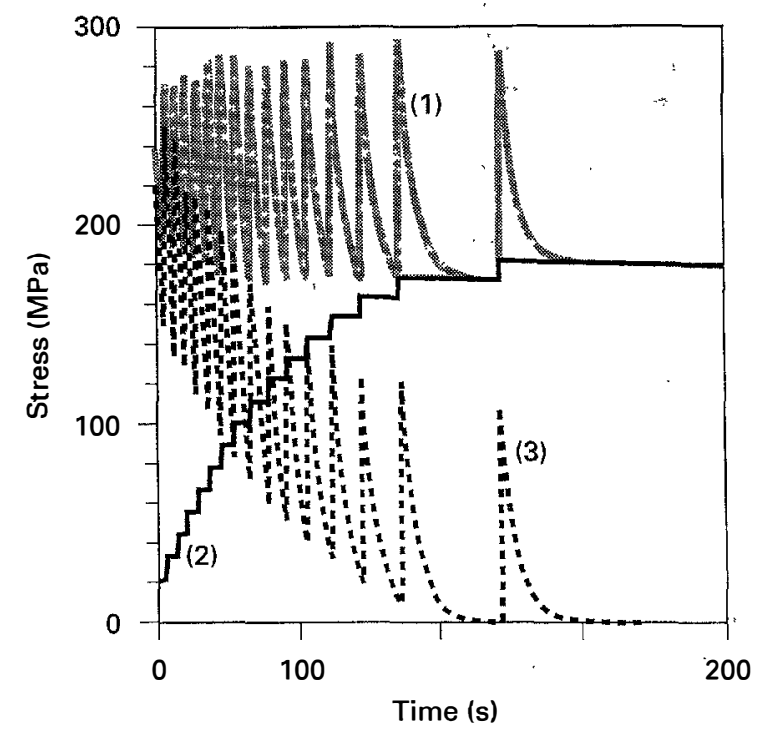

Figure 18 Second set of coefficients: computed breakdown of stress in the step relaxation experiment: (1) over-all stress $\sigma_{z z}$, (2) elastoplastic mechanism part $\sigma_{p z z},(3)$ viscous part $\sigma_{v z z}$.

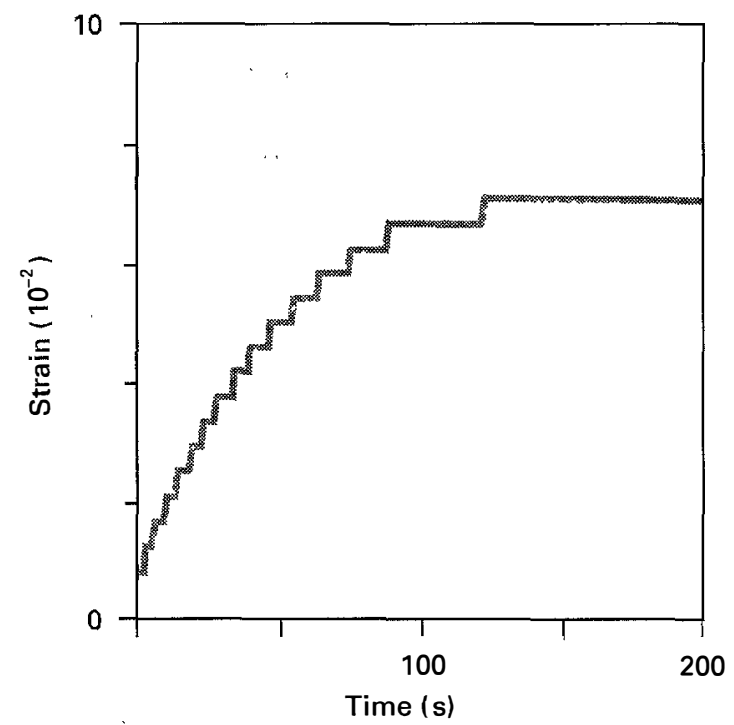

Figure 19 Second set of coefficients: strain evolution. terminates in a corresponding time of $200 \mathrm{~s}$ : this is a consequence of some inappropriate choice for $\eta$, but we had no experimental data to refine this value. Another effect is that the corresponding number of cycles is also lower than could be expected. Fig. 19 shows the evolution of strain, and indicates eventually that the strain level of $10 \%$ is not reached during the test. This tentative calculation leads us to think that a determination of the coefficients for high-density polyethylene from the three steps test, may improve the results.

The numerical analysis of the stress relaxation and recovery experiment does not settle new technical difficulties: it is only needed to substitute a strain decay to a strain increase. So we again considered the two previous sets of coefficients. In each case, the complete stress relaxation test is executed and continued with the stress recovery experiment.

With the first set of coefficients, we chose $0.83 \times 10^{-2} \mathrm{~s}^{-1}$ as the strain decay rate, $\sigma_{1}=$ 3.0 $\mathrm{MPa}$ and $\Delta \sigma_{1}=0.5 \mathrm{MPa}$. From Figs 20 and 21

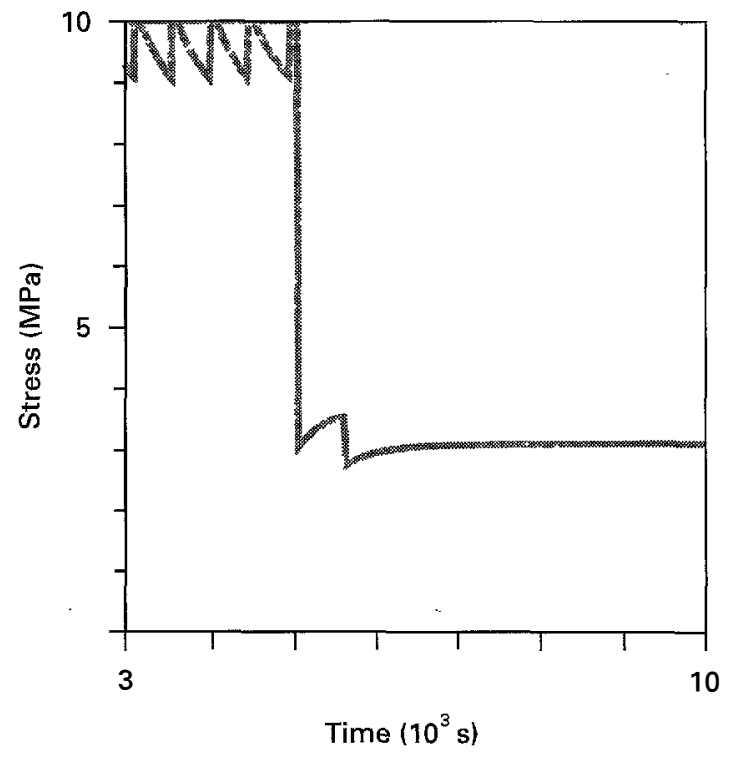

Figure 20 First set of coefficients: stress relaxation and recovery.

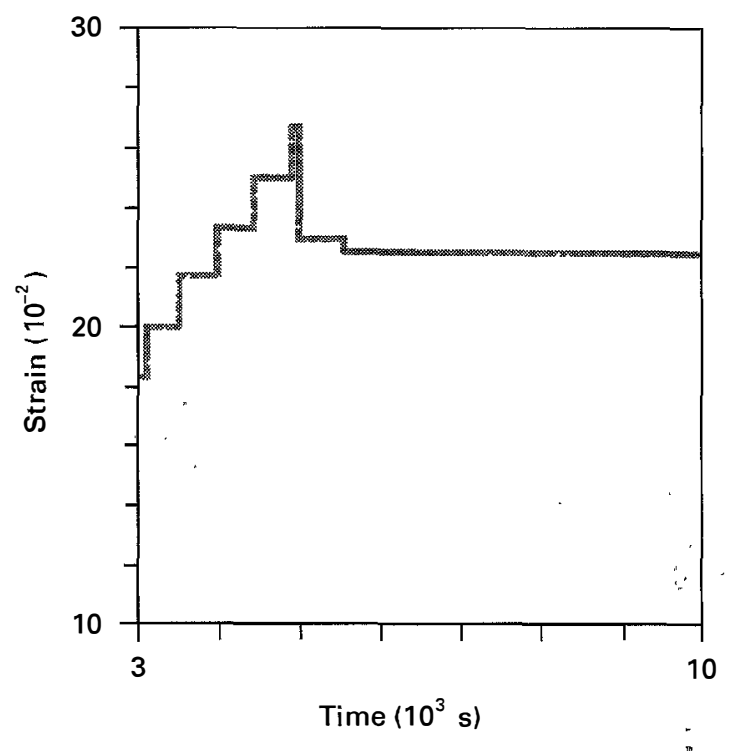

Figure 21 First set of coefficients: strain evolution during stress relaxation and recovery. 


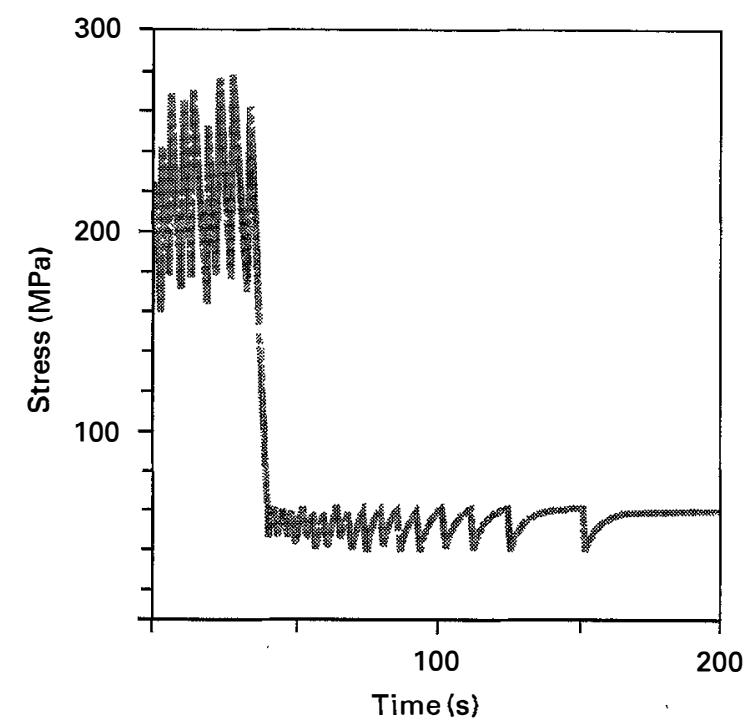

Figure 22 Second set of coefficients: stress relaxation and recovery.

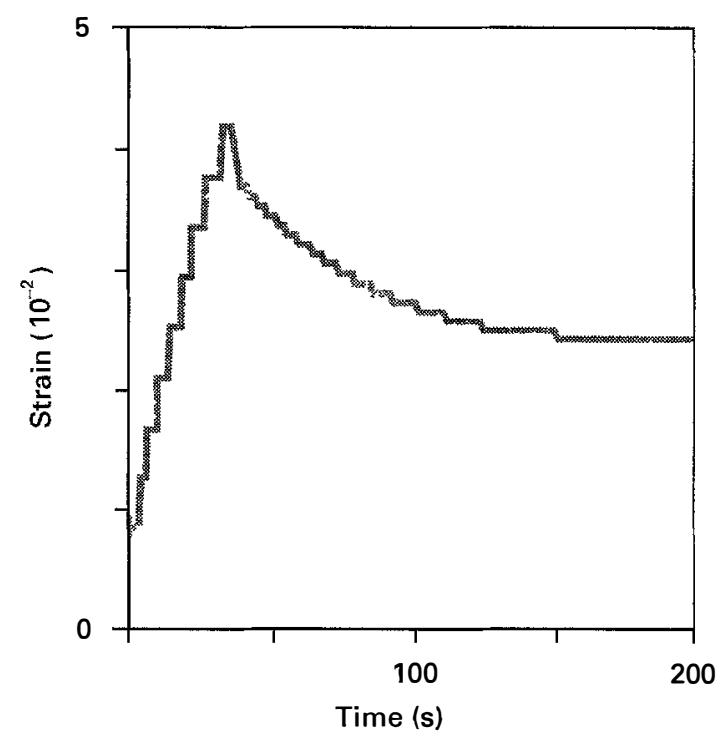

Figure 23 Second set of coefficients: strain evolution during stress relaxation and recovery.

we observe the occurrence of a unique cycle. With the second set of coefficients, we took $0.16 \times 10^{-2} \mathrm{~s}^{-1}$ as the strain decay rate, $\sigma_{1}=50.0 \mathrm{MPa}$ and $\Delta \sigma_{1}=$ 10.0 MPa. This time the number of cycles is more important and the stress stabilizes for a strain level close to $2 \%$ (Figs 22 and 23). Both calculations have to be handled carefully, as no experimental reference can be given, even for the number of cycles.

Incidentally, it should be noted that each simulation approximately takes $1 \mathrm{~h} \mathrm{CPU}$ on a Hewlett-Packard 420 workstation.

Finally, we may conclude from all these results that our modelling enables practical material testing and provides predictive and versatile information concerning the behaviour of the specimen.

\section{Three-dimensional cyclic experiment}

Superiority of a finite-element software becomes evident when we try to analyse successive loading of

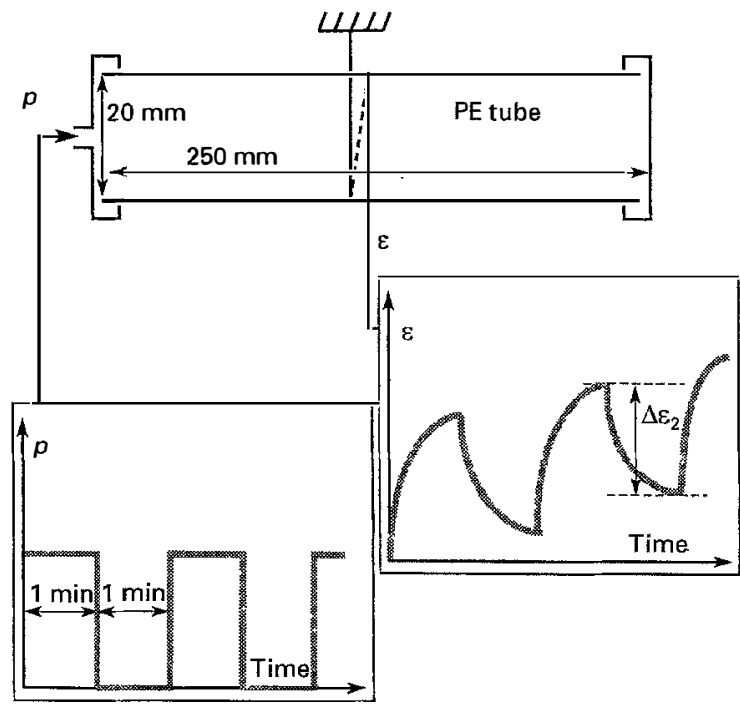

Figure 24 The EAHP experiment.

a real structure. The experiment presented as an example is currently being performed by Professor Pixa's team at the Ecole d'Application des Hauts Polymères in Strasbourg. Let us consider a portion of a polyethylene pipe, about $250 \mathrm{~mm}$ long, $20 \mathrm{~mm}$ diameter and $2 \mathrm{~mm}$ thick. An extremity of the tube is kept fixed and closed. At the other end, a valve enables the admittance and evacuation of pressurized air. This valve electromechanically controls the opening of a fluid reserve bottle so that it is possible to impose on the polyethylene pipe any level of pressure within the range $0.4-20.0 \mathrm{MPa}$ and to hold it as long as is necessary. A thin wire is wound round the specimen and is attached to an extensometer in order to record variations of the diameter and hence deduce radial strain. One test begins by subjecting the tube to a pressure level $p$ (reached in a few seconds) and holding it for 1 minute, then emptying the tube and allowing recovery for 1 minute: the whole scheme is repeated ten times (Fig. 24). For the $i$ th step $\Delta \varepsilon_{\mathrm{i}}$ denotes the amplitude between the maximal and minimal strain. The difference $\Delta \varepsilon_{10}-\Delta \varepsilon_{2}$ is denoted $\Delta \varepsilon$. When the test is repeated with increasing values of $p$, a $\Delta \varepsilon$ versus $p$ curve is drawn. As expected, the tube explodes during the experiment, and this event is announced by a strong change in slope of the previous curve. We simulated the whole test for a section of a tube in the approximation of plane strain. A quarter of the section was meshed with 60 rectangular elements. It is possible to evaluate the radial strain evolution for some values of $p$ (Fig. 25), and to try to predict the $\Delta \varepsilon$ versus real wall stress curve (Fig. 26). In the latter, some noticeable difference between experimental and simulated values are noted at the beginning, but the variation of slopes corresponds quite satisfactorily around $p=10.0 \mathrm{MPa}$. Furthermore, from other calculations, the coefficient of the model responsible for this effect seems to be the threshold $\sigma_{\mathfrak{p}}^{\mathrm{c}}$. The coefficients used in this simulation are as follows (the tensile specimen is made from a different material): $E_{\mathrm{p}}=70.0 \mathrm{MPa}, \quad \sigma_{\mathrm{c}}^{\mathrm{p}}=7.05 \mathrm{MPa}, \quad \alpha=25.03 \mathrm{MPa}$, $E_{\mathrm{v}}=100.0 \mathrm{MPa}, \eta=4.0$ GPas. 


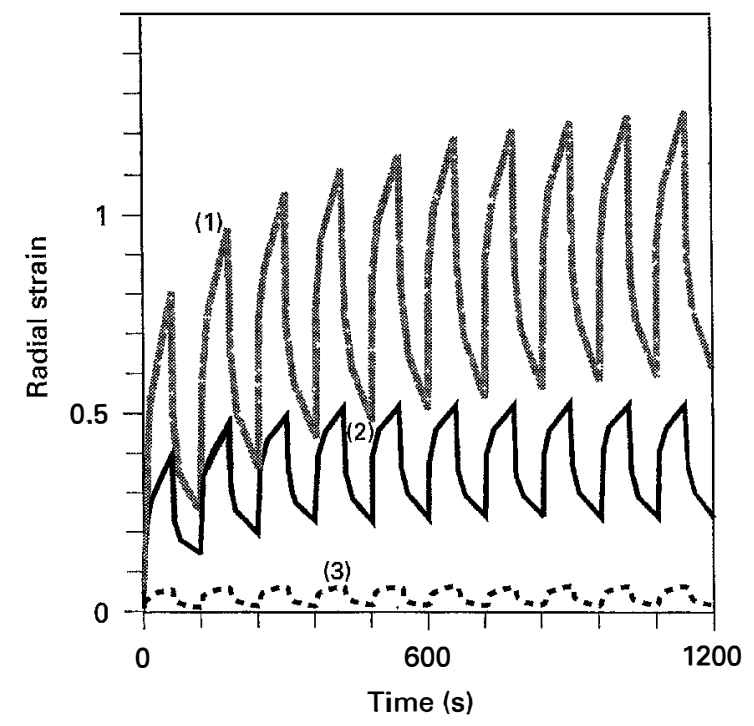

Figure 25 The predicted strain evolution versus time for various levels of imposed pressure (1) $1.2 \mathrm{MPa}$, (2) $0.6 \mathrm{MPa}$, (3) $0.1 \mathrm{MPa}$.

\section{Conclusion}

Two dissipative mechanisms models are capable of predicting the non-linear viscoelastic behaviour of polyethylene. For medium-density polyethylene, we proved that our proposal associating a Maxwell mechanism and an elastoplastic one, was realistic, because the coefficients can be identified from a single three-step uniaxial test, and was practical because it is consistent with literature and enables finite-element simulation of real structures. It is then worthwhile to examine the influence of ageing or temperature to develop this approach, and to try to expand it to a wider set of materials.

\section{Acknowledgements}

The authors thank Dr G. Gary (L.M.S.) who made the versatile data acquisition software agnes available,

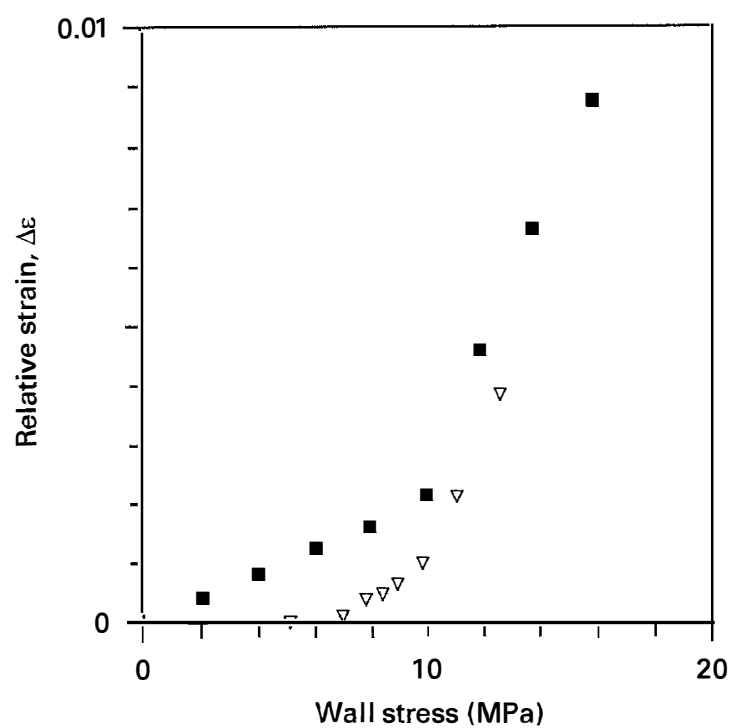

Figure 26 The relative variation of strain $\Delta \varepsilon$ versus real wall stress. Comparison between $(\nabla)$ experimental data and $(\boldsymbol{D})$ numerical simulation.

and gratefully acknowledge financial support from Gaz de France, DETN-CERSTA.

\section{References}

1. J. SWEENEY, and I. M. WARD, J. Mater. Sci. 25 (1990) 697.

2. J.-M. LEFEBVRE and B. ESCAIG, ibid. 20 (1985) 438.

3. D. W. HADLEY and I. M. WARD, in "Encyclopedia of Polymer Science and Engineering", Vol. 9, 2nd Edn (Wiley, New York, 1987) pp. 379-466.

4. J. D. WEBER, in "Conférence du Groupe Français de Rhéologie", 1 March 1972, Metz, Faculté des sciences, edited by Groupe Français de Rhéologie, ISSN 1149, Paris.

Received 11 December 1992

and accepted 4 October 1995 